Louisiana State University

LSU Digital Commons

2-28-2006

\title{
Suppressed reflectivity due to spin-controlled localization in a magnetic semiconductor
}

\author{
F. P. Mena \\ University of Groningen \\ J. F. DiTusa \\ Louisiana State University \\ D. Van Der Marel \\ University of Groningen \\ G. Aeppli \\ University College London \\ D. P. Young \\ Louisiana State University
}

See next page for additional authors

Follow this and additional works at: https://digitalcommons.Isu.edu/physics_astronomy_pubs

\section{Recommended Citation}

Mena, F., DiTusa, J., Van Der Marel, D., Aeppli, G., Young, D., Damascelli, A., \& Mydosh, J. (2006).

Suppressed reflectivity due to spin-controlled localization in a magnetic semiconductor. Physical Review B - Condensed Matter and Materials Physics, 73 (8) https://doi.org/10.1103/PhysRevB.73.085205

This Article is brought to you for free and open access by the Department of Physics \& Astronomy at LSU Digital Commons. It has been accepted for inclusion in Faculty Publications by an authorized administrator of LSU Digital Commons. For more information, please contact ir@lsu.edu. 


\section{Authors}

F. P. Mena, J. F. DiTusa, D. Van Der Marel, G. Aeppli, D. P. Young, A. Damascelli, and J. A. Mydosh 


\title{
archive ouverte UNIGE
} http://archive-ouverte.unige.ch

Article

Suppressed reflectivity due to spin-controlled localization in a magnetic semiconductor

\author{
MENA, F., et al.
}

MENA, F., et al. Suppressed reflectivity due to spin-controlled localization in a magnetic semiconductor. Physical Review. B, Condensed Matter, 2006, vol. 73, no. 8, p. 085205

DOI : 10.1103/PhysRevB.73.085205 


\title{
Suppressed reflectivity due to spin-controlled localization in a magnetic semiconductor
}

\author{
F. P. Mena,,${ }^{1, *}$ J. F. DiTusa, ${ }^{2}$ D. van der Marel,,${ }^{3,1}$ G. Aeppli, ${ }^{4}$ D. P. Young, ${ }^{2}$ A. Damascelli, ${ }^{5}$ and J. A. Mydosh ${ }^{6}$ \\ ${ }^{1}$ Materials Science Centre, University of Groningen, 9747 AG Groningen, The Netherlands \\ ${ }^{2}$ Department of Physics and Astronomy, Louisiana State University, Baton Rouge, Louisiana 70803, USA \\ ${ }^{3}$ Département de Physique de la Matière Condensée, Université de Genève, CH-1211 Genève 4, Switzerland \\ ${ }^{4}$ London Centre for Nanotechnology and Department of Physics and Astronomy, University College London, \\ London WC1E6BT, United Kingdom \\ ${ }^{5}$ Department of Physics \& Astronomy, University of British Columbia, Vancouver, British Columbia V6T 1Z1, Canada \\ ${ }^{6} I I$. Physikalisches Institut, Universität zu Köln, Zülpicher Strasse 77, 5093 Köln, Germany
}

(Received 28 November 2005; published 17 February 2006)

\begin{abstract}
The narrow gap semiconductor FeSi owes its strong paramagnetism to electron-correlation effects. Partial Co substitution for Fe produces a spin-polarized doped semiconductor. The spin polarization causes suppression of the metallic reflectivity and increased scattering of charge carriers, in contrast to what happens in other magnetic semiconductors, where magnetic order reduces the scattering. The loss of metallicity continues progressively even into the fully polarized state, and entails as much as a $25 \%$ reduction in average mean-free path. We attribute the observed effect to a deepening of the potential wells presented by the randomly distributed Co atoms to the majority spin carriers. This mechanism inverts the sequence of steps for dealing with disorder and interactions from that in the classic Al'tshuler Aronov approach-where disorder amplifies the Coulomb interaction between carriers - in that here, the Coulomb interaction leads to spin polarization which in turn amplifies the disorder-induced scattering.
\end{abstract}

DOI: 10.1103/PhysRevB.73.085205 PACS number(s): 78.20.Ci, 71.27.+a, 72.25.Dc, 78.30.-j

\section{INTRODUCTION}

Future technologies based on the control and state of electron spins rather than charges are commonly referred to as spintronics. Efforts to produce materials for spintronics have mostly focused on thin film III-V semiconductors alloyed with manganese. ${ }^{1-4}$ In $(\mathrm{Ga}, \mathrm{Mn})$ As, the most fully characterized of these alloys, Mn substitutes a trivalent $\mathrm{Ga}$ ion and acts as a shallow acceptor just above the valence band. The $\mathrm{Mn}^{2+}$ impurities have a local moment associated with a high spin $(S=5 / 2)$ configuration, and are ferromagnetically coupled below the Curie temperature $\left(T_{C}\right)$ by a small number of itinerant hole carriers. In metallic and ferromagnetic $(\mathrm{Ga}, \mathrm{Mn})$ As these doped holes are thought to reside in an itinerant Mn-derived impurity band ${ }^{5,6}$ about $0.1 \mathrm{eV}$ above the valence band.

Another route to magnetic semiconductors relies on carrier doping into narrow band, strongly correlated insulators. Perhaps the most celebrated is the monosilicide FeSi, which has been investigated for several decades because it has a large $300 \mathrm{~K}$ response to magnetic fields that vanishes as $T$ approaches zero. ${ }^{7-11}$ Together with $\mathrm{CoSi}$ and the unusual metal $\mathrm{MnSi},{ }^{12,13} \mathrm{FeSi}$ belongs to the larger group of transition metal monosilicides, allowing chemical substitutions across the entire series without change in the cubic $B-20$ crystal structure or the nucleation of second phases. ${ }^{7,9}$ Bulk single crystals can be grown and $\mathrm{FeSi}$ can be made metallic and ferromagnetic by the substitution of Co to form the silicon-based magnetic semiconductor $\mathrm{Fe}_{1-y} \mathrm{Co}_{y} \mathrm{Si}^{7,9} \mathrm{Be}-$ cause the metals that result are disordered, quantum interference and electron-electron interactions were found to dominate the temperature and magnetic field dependent dc carrier transport. ${ }^{7}$ Given that optical properties are a key to potential spintronic applications ${ }^{14}$ and have been predicted, but not yet observed, to be sensitive to these interaction effects, ${ }^{15-17}$ we have measured the optical reflectivity $R(\omega)$ of $\mathrm{Fe}_{1-y} \mathrm{Co}_{y} \mathrm{Si}$. Here we present the experimental manifestation of spinpolarization controlled localization on $R(\omega)$ and the optical conductivity $\sigma(\omega)$ of this magnetic semiconductor.

Before presenting the data, we describe what is expected in the three cases of undoped and doped semiconductors, and the simple magnetic metals formed from doped semiconductors. For clean semiconductors, the low-temperature optical conductivity is dominated by excitations across a gap between valence and conduction bands. Warming produces holes in the valence band as well as electrons in the conduction band, adding a Drude peak centered at zero energy to the interband transitions. Chemical doping [Fig. 1(a)] adds carriers to the valence, conduction, or impurity bands and yields a zero-temperature $\sigma(\omega)$ which is very similar to that obtained by warming in the undoped case- the dominant lowenergy feature is a Drude peak with weight proportional to the carrier density $(n)$ and width $1 / \tau$ measuring the scattering rate of the carriers. If the carriers were spin polarized, either via application of an external magnetic field or an internal exchange splitting from spontaneous magnetic order, their band would be split into majority and minority spin bands as in Fig. 1(b). Eventually the minority band can be shifted above the Fermi energy $\left(E_{F}\right)$ resulting in a redistribution of all the carriers into the majority band. The outcome is called a half metallic ferromagnet ${ }^{18}$ [Figs. 1(b) and 1(d)], because electrons carrying the majority spin belong to a partially filled, metallic band, while those carrying the minority spin belong to an empty (at $T=0$ ) conduction band. To accommodate all of the itinerant electrons $E_{F}$ shifts upward, decreasing the density of states (DOS) at $E_{F}$ by a factor of 


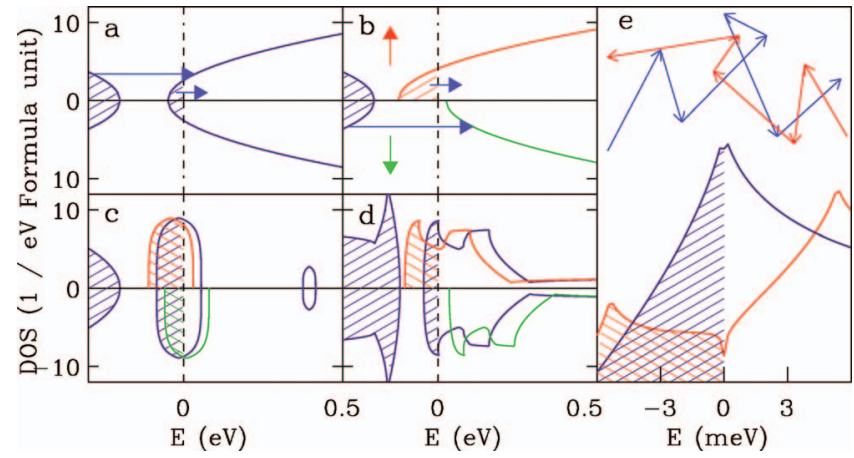

FIG. 1. (Color) Schematic density of states (DOS) for the up and down spin states of semiconductors, ferromagnetic semiconductors, and ferromagnetic metals. Blue arrows depict optically allowed interband transitions: (a) electron doped semiconductor, (b) fully spin-polarized doped semiconductor, (c) doped semiconductor where the added carriers reside in an impurity band within the band gap such as is thought to be the case for $(\mathrm{GaMn}) \mathrm{As}$, (d) fully spinpolarized $\mathrm{Fe}_{0.8} \mathrm{Co}_{0.2} \mathrm{Si}$. (e) Disordered conductor where increased Coulomb interactions lead to an enhanced DOS in the paramagnetic state (purple curve) and a singular, depleted DOS at $E_{F}$ for the spin-polarized state (orange curve). The red and the blue lines at the top of the figure represent a diffusive path for two carriers in a disordered metal. Each crossing represents an interaction event, indicating multiple electron-electron scattering of the same two carriers without breaking quantum coherence.

$2^{2 / 3}$. Naively one might expect this reduction in the DOS to reduce the low-frequency $\sigma(\omega)$. However, the optical sum rule states that the integral of $\sigma(\omega)$ over $\omega$ measures the carrier density $n$, so that conservation of $n$ in the parabolic band leads to conservation of $\sigma(\omega)$ through the phase transition as long as the effective mass of the carriers does not change.

This is precisely what has been observed in previous measurements of spin-polarized metals such as $\mathrm{CrO}_{2}$ and $\mathrm{NiMnSb}$, where the only consequence of the half-metallicity is a slight increase in the scattering rate at energies above that required to transfer a carrier between the majority and the unoccupied minority bands. ${ }^{19,20}$ Similarly, measurements of $\sigma(\omega)$ of the magnetic semiconductor (Ga,Mn)As reveal changes to the spectrum below $T_{C}$ due to a reduction of $\tau^{-1}$ and $m^{*}{ }^{5,6}$ As a result $\sigma(\omega)$ increases at $\omega$ below $350 \mathrm{meV}$, causing an increase of the reflectivity or, in other words, a positive magnetic-order-induced reflectivity. Perhaps the most impressive increases in reflectivity at a ferromagnetic transition occur in the optical spectra of $\mathrm{EuB}_{6}$ and $\mathrm{La}_{1-x} \mathrm{Sr}_{x} \mathrm{MnO}_{3} .{ }^{21,22}$ In these cases cooling into the FM state is accompanied by large increases in the Drude spectral weight due to concurrent semimetal or insulator-metal transitions.

\section{EXPERIMENTAL METHODS}

Single crystals were grown from high purity starting materials $(99.995 \%$ or greater) by either vapor transport, light image furnace floating zone, or modified tri-arc Czochralski methods. X-ray spectra showed all samples to be single

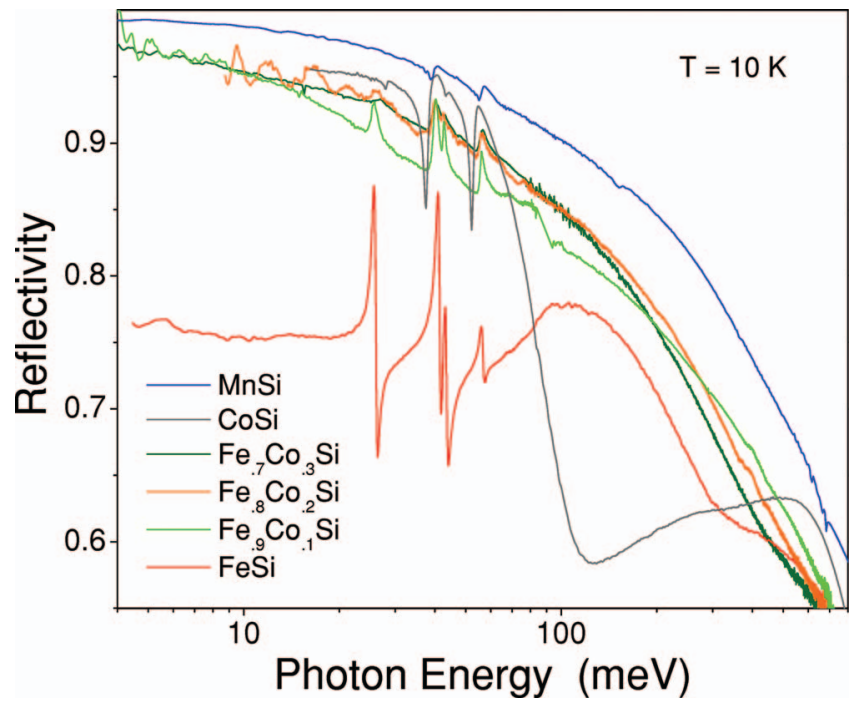

FIG. 2. (Color) Normal incidence reflectivity spectra of FeSi, $\mathrm{CoSi}, \mathrm{Fe}_{1-y} \mathrm{Co}_{y} \mathrm{Si}$, and $\mathrm{MnSi}$ measured at $10 \mathrm{~K}$.

phase with a lattice constant linearly dependent on $y$ demonstrating that Co successfully replaces Fe over the entire concentration range $(0 \leqslant y \leqslant 1)$. Energy dispersive $\mathrm{x}$-ray microanalysis yielded results consistent with the nominal concentrations.

The reflectivity of these single crystals was measured from $4 \mathrm{meV}$ to $0.75 \mathrm{eV}$ while ellipsometry was used to measure directly the dielectric function from 0.74 to $4.5 \mathrm{eV}$. In order to monitor in detail the change of reflectivity as a function of temperature reported in this paper and in a number of other recent experiments of the same laboratory, ${ }^{23-25}$ typical standard optical tail cryostats which are commercially available cannot be used. The precision and stability needed in this work requires a sampling of infrared and optical spectra with a very dense interval of temperatures, and an overall system stability on the order of $0.1 \%$ of the reflected signal or better. We use special design optical cryostats, which dif-

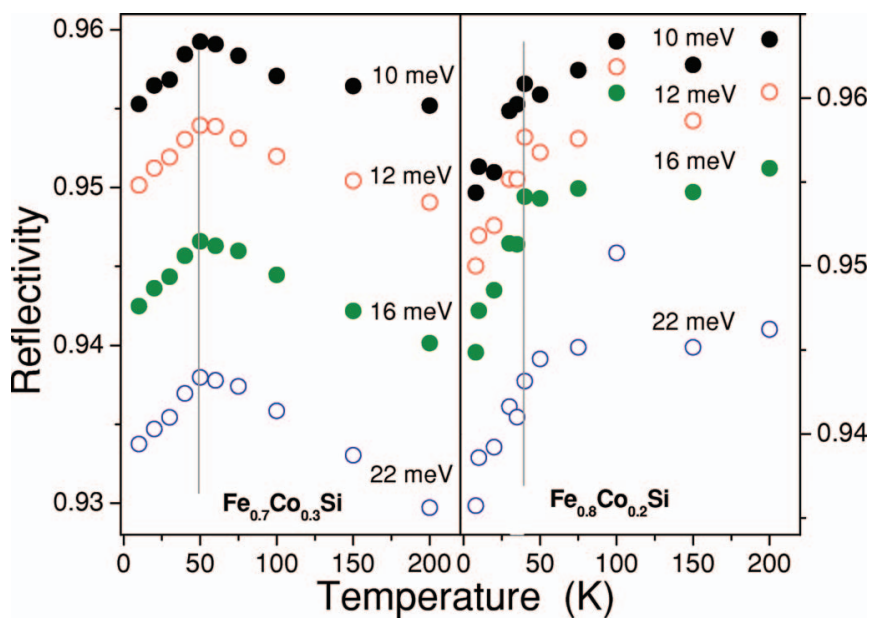

FIG. 3. (Color) Temperature dependence of the reflection coefficient of $\mathrm{Fe}_{0.8} \mathrm{Co}_{0.2} \mathrm{Si}$ and $\mathrm{Fe}_{0.7} \mathrm{Co}_{0.3} \mathrm{Si}$ for four different infrared photon energies. The vertical gray lines indicate the corresponding magnetic ordering temperature. 
fer from commercial designs in several important aspects. Further details are described in Ref. 26.

Between 4 and $750 \mathrm{meV}$ we used Kramers-Kronig relations, along with a Hagen-Rubens extrapolation of $R(\omega)$ data to $\omega=0$, to obtain the phase of $R(\omega)$, and subsequently $\epsilon(\omega)$. $\sigma(\omega)$ is obtained via the relation $\operatorname{Re} \sigma=(\omega / 4 \pi) \operatorname{Im} \epsilon(\omega)$. We have carefully checked that $\sigma(\omega)$ is not significantly altered by our choice of high- and low- $\omega$ terminations. The Kramers-Kronig output was locked to the ellipsometrically measured $\epsilon_{1}(\omega)$ and $\epsilon_{2}(\omega)$, which strongly improves the accuracy. The optical reflectivity at low frequencies and the optical conductivity obtained from the Kramers-Kronig analysis proved to be consistent with the experimental dc resistivity.

\section{OPTICAL REFLECTIVITY}

$\mathrm{Fe}_{1-y} \mathrm{Co}_{y} \mathrm{Si}$ spans insulating, metallic and polarized metallic regimes, and is interesting and important because on the Fe-rich side of the phase diagram ${ }^{7}$ it defies the standard expectations for all three. In Fig. 2 we compare the reflectivity spectra at $10 \mathrm{~K}$ of $\mathrm{FeSi}, \mathrm{CoSi}, \mathrm{MnSi}$, and $\mathrm{Fe}_{1-y} \mathrm{Co}_{y} \mathrm{Si}$ with $y=0.1,0.2,0.3$. The latter three samples order magnetically at $T_{C}=10,36$, and $49 \mathrm{~K}$, while MnSi orders at $29.5 \mathrm{~K}^{7,27}$ All samples shown except $\mathrm{FeSi}$ become $100 \%$ reflecting in the limit $\omega \rightarrow 0$, as expected because they are good electrically conducting materials. The reflectivity spectra reveal optical phonons at approximately $25,40,43$, and $56 \mathrm{meV}$, the shape of which vary progressively from the asymmetric dispersive variety for the semiconducting samples to negative dips for the more metallic samples. This is a direct manifestation of the negative term contributed to the dielectric function by the free charge carriers, in addition to the optical phonon oscillators. $\mathrm{MnSi}$ is a relatively good metal at these temperatures, causing the strong screening of the optical phonons in the reflectivity spectra. In an earlier publication the infrared reflectivity of MnSi has been reported to increase when it becomes magnetically ordered. ${ }^{12}$ This is a natural consequence of the suppression of the spin-disorder scattering of the electrons, simultaneously suppressing the scattering rate of the free charge carriers and increasing the amplitude of the lowfrequency dielectric constant.

The central experimental result of this paper is the temperature dependence of the reflectivity of $\mathrm{Fe}_{1-y} \mathrm{Co}_{y} \mathrm{Si}$, shown in Fig. 3. The different behavior of the paramagnetic state also exists for the transport properties, and it reflects the closer proximity to the metal insulator transition of samples with a smaller carrier concentration. Both $\mathrm{Fe}_{0.8} \mathrm{Co}_{0.2} \mathrm{Si}$ and $\mathrm{Fe}_{0.7} \mathrm{Co}_{0.3} \mathrm{Si}$ behave exactly opposite to $\mathrm{MnSi}$ when the magnetic order occurs: The reflectivity of both samples is clearly suppressed in the ordered state, i.e., in the magnetically ordered phase the material becomes less metallic as compared to the paramagnetic phase. We observed no spin-orderinduced change of reflectivity in the $10 \%$ doped sample, which is not surprising: $T_{C}$ of this sample is $10 \mathrm{~K}$. Assuming that the spin-ordering induced changes are similar to those of the other two samples, the estimated change of reflectivity between $6 \mathrm{~K}$ (the lower limit of this cryostat) and $10 \mathrm{~K}$ is only about 0.0005 , which is too small to detect with the current accuracy.

\section{OPTICAL CONDUCTIVITY}

In order to obtain a clear experimental picture of this effect, we examine the optical conductivity spectra for a few selected temperatures, shown in Fig. 4 for the semiconducting parent compound $\mathrm{FeSi}$ and $\mathrm{Fe}_{1-y} \mathrm{Co}_{y} \mathrm{Si}$. We begin with pure CoSi (top frames in Fig. 4), long known as a diamagnetic metal with a very low carrier density, $\sim 1 \%$ of electrons/formula unit. ${ }^{28}$ Our data agree with the simple ideas outlined above; i.e., there is a small Drude peak centered at $\omega=0$ which coexists with interband transitions beginning at $\sim 125 \mathrm{meV}$. At $T=10 \mathrm{~K}$, the width of the Drude peak is $\hbar / \tau=20 \mathrm{meV}$, which in the simplest analysis implies a carrier mean free path of $5 \mathrm{~nm}$. The main effect of warming to $300 \mathrm{~K}$ is to broaden the Drude peak by an amount less than $k_{B} T$.

The lower frame of Fig. 4 displays data for the alloy's other end member, insulating FeSi. The optical conductivity, which shows no hint of a band gap at $300 \mathrm{~K}$, is almost completely suppressed below $75 \mathrm{meV}$ upon cooling to $10 \mathrm{~K}$, as in Ref. 29 which emphasized that in a conventional band picture, remnants of a $60 \mathrm{meV}$ gap should be clearly visible at $300 \mathrm{~K}(26 \mathrm{meV})$. This disappearance of the semiconductor gap at approximately $200 \mathrm{~K}$ has been confirmed by subsequent investigations of the optical properties. ${ }^{30,31} \mathrm{~A}$ second important feature is that the energy range over which $\sigma(\omega)$ changes as a function of temperature, is extremely large as indicated by the logarithmic $\omega$ scale in Fig. 4. Careful analysis of the temperature dependence of the integrated spectral weight ${ }^{32}$ confirms the result of Ref. 29, that there is a lack of spectral weight conservation to energies above 80 times $E_{g}$. Even if the spectral weight would be distributed over a wide spectral range in an unmeasurable way, it is not recovered on the energy scale of a few times the gap as expected.

While the obliteration of all remnants of a $60 \mathrm{meV}$ gap at $300 \mathrm{~K}$ can be attributed, at least in part, to the feedback of vibrational disorder on the electronic structure using a conventional band picture where electron-electron correlations are treated within the framework of the local density approximation, ${ }^{33}$ the issue of the spectral weight redistribution appears to require a more rigorous treatment of the correlation effects. Urasaki and $\mathrm{Saso}^{34}$ treated the bands on either side of the gap with the two-band Hubbard model, while assuming a moderate $U(U=0.5 \mathrm{eV})$. Their calculated optical spectra agree in detail with the experiments. In particular their model explains both the temperature shift of gap edge, and the temperature dependent spectral weight redistribution. Taken together this suggests the important role of electroncorrelations for the physical properties of $\mathrm{FeSi}^{34-37}$

Doping FeSi via substitution of Co for Fe yields similar problems for the standard model underlying semiconductor optics. The conductivity of the $10 \%$ doped sample exhibits a suppression below $10 \mathrm{meV}$ for $T<100 \mathrm{~K}$, but there is no complete gap as in $\mathrm{FeSi}$ at the same temperatures. In contrast to the data for pure $\mathrm{CoSi}$, but in agreement with our findings for $\mathrm{FeSi}$ at $300 \mathrm{~K}, \mathrm{Fe}_{1-y} \mathrm{Co}_{y} \mathrm{Si}$ for the three dopings consid- 


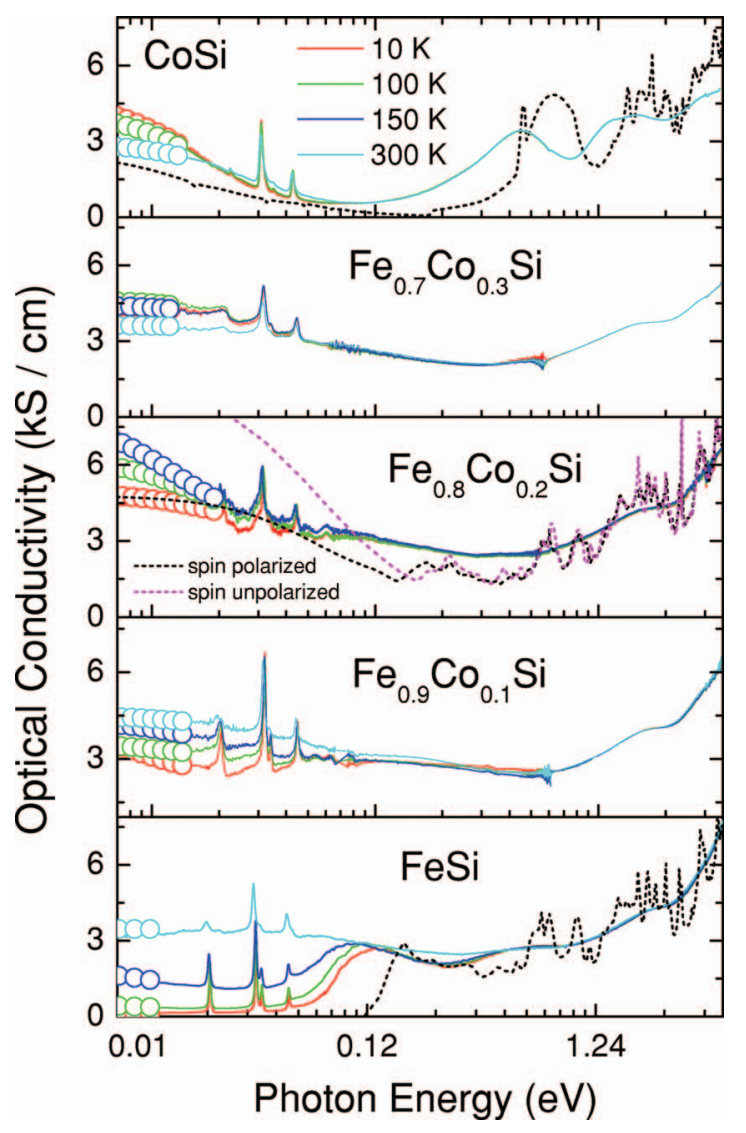

FIG. 4. (Color) Experimental optical conductivity spectra of $\mathrm{Fe}_{1-y} \mathrm{Co}_{y} \mathrm{Si}$ for $y=0.0,0.1,0.2,0.3$, and 1.0 (solid lines and open circles). The calculated optical conductivity using the local density approximation is shown for $\mathrm{FeSi}, \mathrm{Fe}_{0.8} \mathrm{Co}_{0.2} \mathrm{Si}$, and $\mathrm{CoSi}$ (dashed curves). Widths $\left(\tau^{-1}\right)$ of the Drude peaks were chosen to minimize the deviation from the experiment spectra. For $\mathrm{Fe}_{0.8} \mathrm{Co}_{0.2} \mathrm{Si}$, the calculation corresponding to full spin polarization (olive dashed curves) is a much better estimate for the Drude weight than unpolarized calculation (purple dashed curve), even at $300 \mathrm{~K}$, consistent with short-range magnetic order well above $T_{C}$.

ered here displays a $\sigma(\omega)$ which decays weakly from $\hbar \omega$ $=0$ to $350 \mathrm{meV}$, while all traces of the gap in the pure FeSi parent are obliterated. ${ }^{38}$ Even with the assumption of a scattering rate in excess of the $60 \mathrm{meV}$ gap of $\mathrm{FeSi}$, a simple Drude analysis (dashed lines in Fig. 4) based on our band structure calculations cannot account for $\sigma(\omega)$. We conclude that treating the electrons in $\mathrm{Fe}_{1-y} \mathrm{Co}_{y} \mathrm{Si}$ as a simple Fermi liquid formed in the conduction band is incorrect, notwithstanding the remarkable simplicity of aggregate $\omega=0$ properties such as the normal Hall effect and ordered magnetization, which correspond to one carrier and one polarized spin per Co atom. ${ }^{7}$

Beyond showing that the parent insulator and its electrondoped derivative violate standard ideas about undoped and doped semiconductors, Fig. 4 also reveals that $\mathrm{Fe}_{1-y} \mathrm{Co}_{y} \mathrm{Si}$ defies expectations for itinerant magnets. In particular, cooling yields a suppression of the low-frequency conductivity of a different qualitative nature than seen for $\mathrm{FeSi}$, where it occurs throughout the gapped region. The suppression of the optical conductivity is of course consistent with the suppres-

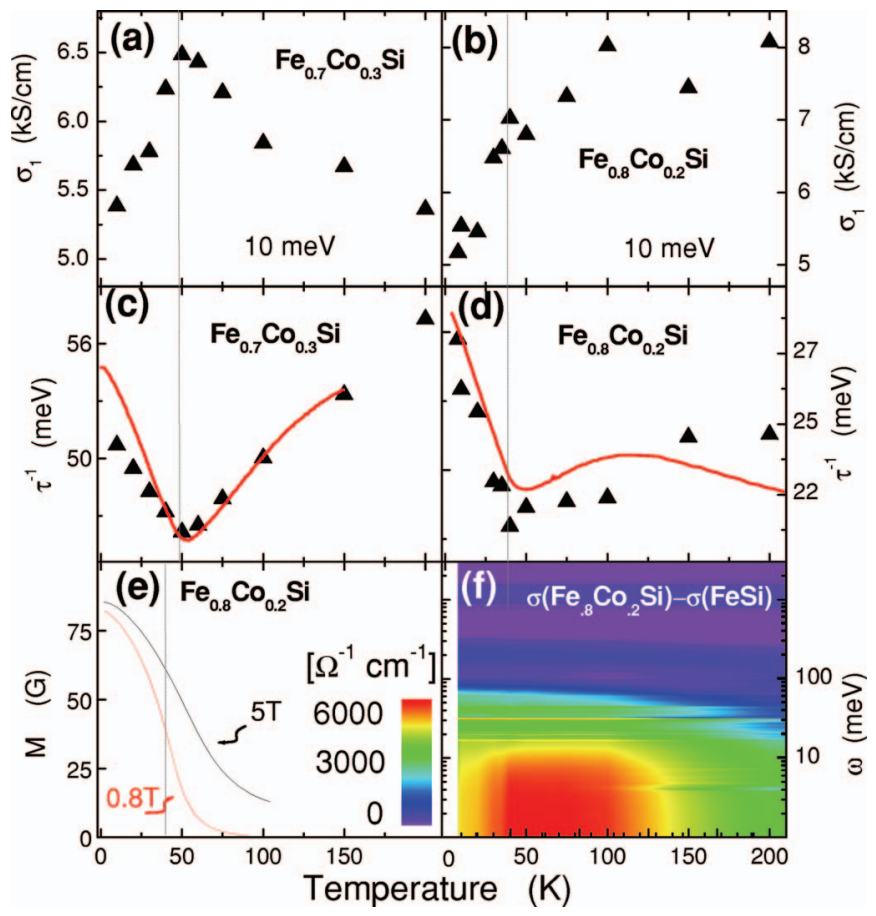

FIG. 5. (Color) Temperature dependence of related physical properties of $\mathrm{Fe}_{1-y} \mathrm{Co}_{y} \mathrm{Si}$ for two different doping concentrations. The optical conductivity $\sigma(\omega)$ for $\hbar \omega=10 \mathrm{meV}$ (a) and (b), the corresponding optical scattering rates $\hbar / \tau$ [triangles in (c) and (d)], $\mathrm{dc}$ resistivities scaled as to overlay the scattering rates [red solid curves in (c) and (d)], bulk magnetization of $\mathrm{Fe}_{0.8} \mathrm{Co}_{0.2} \mathrm{Si}$ (e), and optical conductivity difference of $\mathrm{Fe}_{0.8} \mathrm{Co}_{0.2} \mathrm{Si}$ and $\mathrm{FeSi}$ for a large range of temperatures and frequencies (f). The corresponding magnetic ordering temperatures are indicated by the gray lines.

sion of the dc conductivity from the dc transport measurements, as well as the raw reflectivity data $R(\omega)$ of Fig. 3 . Thus, in contrast to what occurs for all other metallic ferromagnets, including MnSi (Ref. 32) and (Ga,Mn)As, ${ }^{5,6}$ the approach and onset of magnetic order at $T_{C}=36 \mathrm{~K}$ decreases the conductivity and suppresses the metallic screening of $\mathrm{Fe}_{1-y} \mathrm{Co}_{y} \mathrm{Si}$.

Figure 5 reveals more detail on the evolution of the optical data with $T$, and compares them to transport and magnetization data. $R(\omega)$ in Fig. 3 at low $\omega$ simply follows the $\sigma(\omega=0)$ (Fig. 5), which experiences its main drop below $T_{C}$. For higher $\omega$ (not shown), $R(\omega)$ decreases continuously from $300 \mathrm{~K}$, with no visible anomaly at $T_{C}$ for photon energies larger than $40 \mathrm{meV}$. The obliteration of singular behavior near $T_{C}$ with increasing $\omega$ is in accord with the extended critical regime, or superparamagnetism (field-induced shortrange order), indicated by the magnetization data of Fig. 5(d). Here a modest (compared to $k_{B} T$ ) external field of $5 \mathrm{~T}$ produces very appreciable polarization to $T$ 's as high as $100 \mathrm{~K} \sim 3 T_{C}$. Figure $5(\mathrm{f})$ shows $\sigma(\omega, T)$ of $\mathrm{Fe}_{0.8} \mathrm{Co}_{0.2} \mathrm{Si}$ after subtraction of $\sigma(\omega)$ of $\mathrm{FeSi}$, revealing where the added carriers reside in the excitation spectrum of the nominally pure compound. Cooling builds up the differential (relative to the insulator) spectral weight down to $T_{C}$, whereupon there is a loss especially apparent below $25 \mathrm{meV}$. Again, our observation of a reduced spectral weight below $T_{C}$ contradicts both 


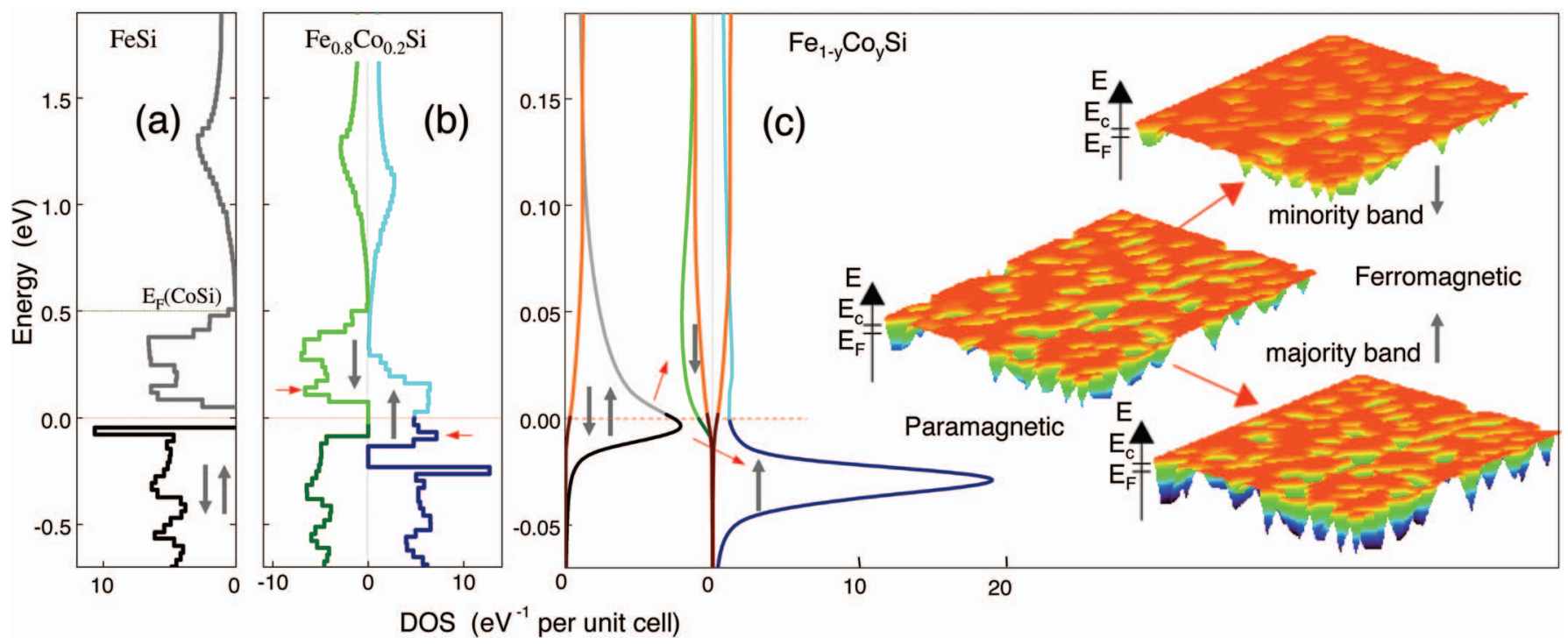

FIG. 6. (Color) (a) Calculated density of states of FeSi. The Fermi energy (red line) is inside the semiconductor gap. The band calculations of CoSi give a very similar DOS, with the Fermi energy shifted to a higher energy (dark dotted line). (b) $\mathrm{DOS}_{\text {of }} \mathrm{Fe}_{0.8} \mathrm{Co}_{0.2} \mathrm{Si}$ using the virtual crystal approximation (Ref. 39). These results confirm the full spin polarization of $\mathrm{Fe}_{0.8} \mathrm{Co}_{0.2} \mathrm{Si}$. Arrows indicate $E_{F}$ in unpolarized state. (c) Local DOS at the Co atom substituting Fe in FeSi in the unpolarized state (gray curves) and the spin-polarized state (blue and green curves), calculated using the tight-binding Green's function formalism (Refs. 40 and 41), while treating the on-site Hubbard repulsion in the mean-field approximation (Refs. 42 and 43). The orange curves are the DOS of the FeSi host material. The Fermi energy $\left(E_{F}\right)$ lies just above the FeSi conduction band edge $\left(E_{c}\right)$. The potential energy landscape for the carriers due to the Co impurities is sketched on the righthand side. In the ferromagnetic state carriers in the majority spin subband scatter more strongly from the random potential than either carriers in the minority spin subband or in paramagnetic bands due to the increased depth of the potential wells.

the standard models based on independent quasiparticles and measurements for other magnetic semiconductors. ${ }^{5,6}$

In addition, cooling below $T_{C}$ causes the wide Drude-like peak to broaden, rather than to narrow. We have parametrized the low- $\omega \sigma(\omega)$ by fitting the standard Drude form, superposed on a $T$-independent Lorentzian peak at $800 \mathrm{~cm}^{-1}$, to the data. Figure 5 displays the resulting scattering rates $\left(\tau^{-1}\right)$, which track the bulk resistivities $\left[1 / \sigma_{1}(\omega=0)\right]$ and undergo a sharp upturn below $T_{C}$. The important and unique contribution of the optical data is to show that the unusual rise in resistivity below $T_{C}$ is due to enhanced scattering as opposed to a reduction of the charge carrier density/mass ratio.

\section{DISCUSSION}

To begin to understand our data, we have calculated the density of states (Fig. 6) as well as $\sigma(\omega)$ (Fig. 4) derived from the band structure of $\mathrm{Fe}_{1-y} \mathrm{Co}_{y} \mathrm{Si}$. These calculations employ the local density approximation (LDA) with the Gunnarsson-Lundquist exchange correlation potential carried out self-consistently using the full potential linear muffin-tin orbital method. ${ }^{44-46}$ For $\mathrm{Fe}_{0.8} \mathrm{Co}_{0.2} \mathrm{Si}$ a noninteger charge to iron in $\mathrm{FeSi}$ was assigned. We evaluated $\sigma(\omega)$ with a $k$-space integration over 216 points in the irreducible part of the Brillouin zone. ${ }^{38}$ The band structure agrees with the magnetization and Hall effect and more complicated supercell calculations, in that the minority spin Fermi surface resides in a gap. ${ }^{7,8,39}$ It also supports the picture that Co doping merely adds electrons to bands inherited from the FeSi parent, re- vealing, as does experiment, that $\mathrm{CoSi}$ (with one extra electron $/ \mathrm{Fe}$ ) is a very low $n$ metal.

While treating electron correlations within the framework of the LDA (Fig. 6) has some successes (for example, in explaining the ground-state properties), for the description of the relevant (magneto) transport and optical properties it is necessary to take into account the impact of electronic correlations on the excited states. Generally speaking this requires a treatment beyond the local density approximation. Important experimental observations which need to be understood are the apparent loss of carriers at low- $\omega$, and the increase-instead of the conventional decrease-in $\tau^{-1}$ below $T_{C}$ of $\mathrm{Fe}_{1-y} \mathrm{Co}_{y} \mathrm{Si}$. To make progress, the Coulomb interactions need to be considered. How these underpin the moment formation at modest $T$, as well as the rapid filling of the gap in the parent compound via the paradigm of the Kondo insulator, is discussed elsewhere. ${ }^{34-37}$

Why do we observe an increased scattering rate below $T_{C}$, opposite in sign to Mn-doped GaAs and MnSi? For weak spin polarization, this effect most likely follows from the interplay of Coulomb coupling and the quantum mechanical interference of diffusing charge carriers. ${ }^{15,16}$ This feature of disordered metals, hitherto seen only in transport and tunneling, but witnessed optically for the first time in our experiments, is demonstrated schematically in Fig. 1(g). Arrows at the top of the figure demonstrate diffusive paths for two carriers in a disordered metal. The large elastic scattering rate results in multiple scattering of these same two carriers without breaking quantum coherence, effectively enhancing their Coulomb interaction because the carriers are visible to each other more often. The increased Coulomb coupling induces 
square-root singularities in the density of states at $E_{F} \cdot{ }^{15,16}$ Spin polarization, either from external magnetic fields or a spontaneous magnetization, shifts the singularities with respect to $E_{F}$ resulting in a reduction of $\sigma(\omega),{ }^{15,16}$ illustrated schematically in the figure. Thus $\sigma(\omega)$ and $R(\omega)$ display singular behavior in $T$ at low $\omega$ just as we observe. $\mathrm{MnSi}$, the only other known magnet in the transition metal monosilicide group, is not a disordered metal, in accordance with our observation of no magnetic-order-induced reduction of the reflectivity. ${ }^{32}$ However, well below $T_{C}$ the saturation magnetization of $\mathrm{Fe}_{1-y} \mathrm{Co}_{y} \mathrm{Si}$ with $x<0.3$ approaches ${ }^{7}$ that of a halfmetallic ferromagnet [Fig. 1(d)]. In this limit one of two spin components is absent near the Fermi energy, a state of affairs quite distant from the situation considered in Fig. 1(g). As we will explain below, disorder and Coulomb interactions appear to control the optical and magnetotransport anomalies of these compounds also in the limit of strong spin polarization.

The explanation here stems from the strong $3 d$ character of the conduction bands. In a tight binding picture of $\mathrm{Fe}_{1-y} \mathrm{Co}_{y} \mathrm{Si}$ the random substitution of $\mathrm{Co}$ for $\mathrm{Fe}$ within the FeSi lattice corresponds to deepening the potential well of the Co sites, allowing one extra electron per site to enter. This attractive potential causes a downward shift of the local DOS of the Co atoms relative to the FeSi DOS. Provided that this potential exceeds a critical value, an impurity state will be pulled below the conduction band, i.e., inside the semiconductor gap. However, even if the potential is smaller than the critical value, the local DOS on the Co sites has a pile up of states near the gap [gray curves in Fig. 6(c)]. In this scenario the elastic scattering of the conduction electrons is a consequence of the disordered potential landscape [Fig. 6(c)] and, in the case of $\mathrm{Fe}_{1-y} \mathrm{Co}_{y} \mathrm{Si}$, brings the system close to an Anderson metal-insulator transition. How close is indicated by the product $k_{F} l$ of the Fermi vector and mean free path which we measure to be 6.9 if we assume a single Fermi surface, and 1.3 if we consider that there are 12 electron pockets. ${ }^{47}$ As this weakly metallic electron gas is cooled, the spin splitting of the bands due to the exchange interaction shifts the majority and minority bands of Co-3d character with respect to the FeSi conduction band. The consequence for the disordered potential landscape is illustrated in the right hand side of Fig. 6(c) where the depth of the potential wells in proximity to the Co sites is increased (decreased) for the majority (minority) spin subband. Consequently, the majority spins are more strongly scattered compared to the paramagnetic, or unpolarized high-temperature, state. The net magnetoresistance of two parallel channels is ${ }^{48} \rho^{-1}=\rho_{\uparrow}^{-1}$ $+\rho^{-1}$, where $\rho_{\sigma}$ are the resitivities of each spin channel separately, if spin flip scattering, such as arising from spin-orbit coupling, can be neglected. In the limit of vanishing spinpolarization the sign of the magnetoresistance depends on the microscopic details of the model describing $\rho_{\sigma}$ as a function of doping. ${ }^{48}$ However, in the present case an almost fully spin-polarized state is observed at low temperatures, hence we consider here the limit where the spin splitting of the energy levels is large. Due to hopping between the Co sites the states inside the gap form bands, and at the high doping levels considered here the width of these bands is a considerable fraction of the bandwidth of FeSi. For moderate spin splitting, these bands merge with the FeSi conduction band, and the DOS at $E_{F}$ remains ungapped, and the density of states still resembles that given by the virtual crystal approximation $^{39}$ presented in Fig. 6(b). Ultimately, for a sufficiently large spin splitting the majority spin states become separated from the conduction band by a gap. Since the number of doped electrons is exactly equal to the number of majority spin states, the Fermi level resides inside this gap. Consequently this state of matter is not only fully spin polarized, but it is also an insulator. In other words, in the limit of large spin polarization both $\rho_{\uparrow}$ and $\rho_{\downarrow}$ diverge, the former because all localized up-states are occupied, the latter because the down states are empty in this limit. While these extreme conditions appear not to be met in the case of Codoped FeSi, the majority spins in this material do outnumber the minority spins by a large fraction below $T_{C}$, hence the dominant effect at high spin polarization is a positive magnetoresistance, just as we observe in Fig. 5.

The Al'tshuler-Aronov description and the model outlined above both take elastic scattering and Coulomb interactions as the starting point, and both lead to the same conclusion, namely, that spin polarization of a disordered metal leads to enhanced scattering. Whereas the former captures the subtle low-energy scale effects caused by the coherent scattering between impurities, the latter becomes relevant in the limit of strong local perturbing potentials and a high degree of spin polarization. Actually, in our description, we consider first the effect of the Coulomb interaction as the cause of spin order and therefore, via exchange splitting of the bands, an amplifier of potential scattering, whereas Al'tshulerAronov considers disorder as source of scattering which then amplifies the Coulomb interaction. The unification of these two limiting cases in a single framework remains a theoretical challenge yet to be met, although we suspect that a twoparameter scaling description might eventually account for the remarkable success of the Al'tshuler-Aronov expressions in accounting for the magnetoconductance of FeCoSi over such a wide range of fields and temperature. ${ }^{7}$

The reason for the differences from $\sigma(\omega)$ of (GaMn)As now becomes clear; the bands with $\mathrm{Mn} d$ character are well below the GaAs valence band and produce a local magnetic moment interacting with the conducting holes. Therefore the effect of polarization on the carrier elastic scattering is less important and only small changes to $\sigma(\omega)$ due to variations in the spin disorder scattering are observed.

\section{CONCLUSIONS}

We have shown that the optical properties of $\mathrm{Fe}_{1-y} \mathrm{Co}_{y} \mathrm{Si}$ are very different from those for $(\mathrm{Ga}, \mathrm{Mn}) \mathrm{As}$, even though bulk properties such as the off-diagonal conductivity are remarkably similar. ${ }^{7,8}$ Doping produces an optical response throughout the gap of FeSi, implying that we are dealing not with an impurity band as in $(\mathrm{Ga}, \mathrm{Mn}) \mathrm{As}$ - but rather with carriers donated to the conduction band of FeSi. Finally, we have discovered an optical reflectivity which decreases rather than increases upon entering the spin-polarized state. The corresponding rise in the scattering rate, uniquely visible in the optical data, demonstrates that the origin of this effect is 
the Coulomb interaction between electrons in a disordered system.

\section{ACKNOWLEDGMENTS}

This work was supported by the National Science Foundation under Contract No. DMR0406140, a Wolfson-Royal Society Research Merit Award, the Basic Technologies pro- gram of the U.K. Research Councils, the Swiss National Science Foundation through the NCCR "Materials with Novel Electronic Properties," and the Netherlands Foundation for Fundamental Research on Matter with financial aid from the Nederlandse Organisatie voor Wetenschappelijk Onderzoek. We gratefully acknowledge A. A. Menovsky, C. Presura, and A. I. Poteryaev for their assistance with crystal growth, optical experiments and LDA calculations, respectively.
*Current address: Space Research Organization of The Netherlands, Groningen, The Netherlands.

${ }^{1}$ H. Ohno, H. Munekata, T. Penney, S. von Molnar, and L. L. Chang, Phys. Rev. Lett. 68, 2664 (1992).

${ }^{2}$ H. Ohno, A. Shen, F. Matsukura, A. Oiwa, A. Endo, S. Katsumoto, and Y. Iye, Appl. Phys. Lett. 69, 363 (1996).

${ }^{3}$ F. Matsukura, H. Ohno, A. Shen, and Y. Sugawara, Phys. Rev. B 57, R2037 (1998).

${ }^{4}$ M. L. Reed, N. A. El-Masry, H. H. Stadelmaier, M. K. Ritums, M. J. Reed, C. A. Parker, J. C. Roberts, and S. M. Bedair, Appl. Phys. Lett. 79, 3473 (2001).

${ }^{5}$ E. J. Singley, R. Kawakami, D. D. Awschalom, and D. N. Basov, Phys. Rev. Lett. 89, 097203 (2002).

${ }^{6}$ E. J. Singley, K. S. Burch, R. Kawakami, J. Stephens, D. D. Awschalom, and D. N. Basov, Phys. Rev. B 68, 165204 (2003).

${ }^{7}$ N. Manyala, Y. Sidis, J. F. DiTusa, G. Aeppli, D. P. Young, and Z. Fisk, Nature (London) 404, 581 (2000).

${ }^{8}$ N. Manyala, Y. Sidis, J. F. DiTusa, G. Aeppli, D. P. Young, and Z. Fisk, Nat. Mater. 3, 255 (2004).

${ }^{9}$ J. H. Wernick, G. K. Wertheim, and R. C. Sherwood, Mater. Res. Bull. 7, 1431 (1972).

${ }^{10}$ V. Jaccarino, G. K. Wertheim, J. H. Wernick, L. R. Walker, and S. Arajs, Phys. Rev. 160, 476 (1967).

${ }^{11}$ G. Aeppli and Z. Fisk, Comments Condens. Matter Phys. 16, 155 (1992).

${ }^{12}$ F. P. Mena, D. van der Marel, A. Damascelli, M. Fäth, A. A. Menovsky, and J. A. Mydosh, Phys. Rev. B 67, 241101(R) (2003).

${ }^{13}$ N. Doiron-Leyraud, I. R. Walker, L. Taillefer, M. J. Steiner, S. R. Julian, and G. G. Lonzarich, Nature (London) 425, 595 (2003).

${ }^{14}$ S. A. Wolf, D. D. Awschalom, R. A. Buhrman, J. M. Daughton, S. von Molnár, M. L. Roukes, A. Y. Chtchelkanova, and D. M. Treger, Science 294, 1488 (2001).

${ }^{15}$ B. L. Al'tshuler, A. G. Aronov, M. G. Gershenson, and Yu. V. Sharvin, Sov. Sci. Rev., Sect. A 9, 223 (1987).

${ }^{16}$ P. A. Lee and T. V. Ramakrishnan, Rev. Mod. Phys. 57, 287 (1985).

${ }^{17}$ A. J. Millis and P. A. Lee, Phys. Rev. B 30, 6170 (1984); 31, 5523.3(E) (1985).

${ }^{18}$ R. A. de Groot, F. M. Mueller, P. G. van Engen, and K. H. J. Buschow, Phys. Rev. Lett. 50, 2024 (1983).

${ }^{19}$ E. J. Singley, C. P. Weber, D. N. Basov, A. Barry, and J. M. D. Coey, Phys. Rev. B 60, 4126 (1999).

${ }^{20}$ F. B. Mancoff, B. M. Clemens, E. J. Singley, and D. N. Basov, Phys. Rev. B 60, R12565 (1999).

${ }^{21}$ L. Degiorgi, E. Felder, H. R. Ott, J. L. Sarrao, and Z. Fisk, Phys. Rev. Lett. 79, 5134 (1997).

${ }^{22}$ Y. Okimoto, T. Katsufuji, T. Ishikawa, A. Urushibara, T. Arima, and Y. Tokura, Phys. Rev. Lett. 75, 109 (1995).
${ }^{23}$ H. J. A. Molegraaf, C. Presura, D. van der Marel, P. H. Kes, and M. Li, Science 295, 2239 (2002).

${ }^{24}$ D. van der Marel, H. J. A. Molegraaf, J. Zaanen, Z. Nussinov, F. Carbone, A. Damascelli, H. Eisaki, M. Greven, P. H. Kes, and M. Li, Nature (London) 425, 271 (2003).

${ }^{25}$ A. B. Kuzmenko, N. Tombros, H. J. A. Molegraaf, M. Grüninger, D. van der Marel, and S. Uchida Phys. Rev. Lett. 91, 037004 (2003)

${ }^{26}$ For details of the cryostat design see optics.unige.ch/vdm/ marel_files/cryostat_design.htm

${ }^{27}$ H. J. Williams, J. H. Wernick, R. C. Sherwood, and G. K. Wertheim, J. Appl. Phys. 37, 1256 (1966).

${ }^{28}$ S. Asanabe, D. Shinoda, and Y. Sasaki, Phys. Rev. 134, A774 (1964).

${ }^{29}$ Z. Schlesinger, Z. Fisk, H. T. Zhang, M. B. Maple, J. F. DiTusa, and G. Aeppli, Phys. Rev. Lett. 71, 1748 (1993).

${ }^{30}$ L. DeGiorgi, M. B. Hunt, H. R. Ott, M. Dressel, B. J. Feenstra, G. Grüner, Z. Fisk, and P. Canfield, Europhys. Lett. 28, 341 (1994).

${ }^{31}$ A. Damascelli, K. Schulte, D. van der Marel, and A. A. Menovsky, Phys. Rev. B 55, R4863 (1997).

${ }^{32}$ F. P. Mena, Ph.D. thesis, University of Groningen, Groningen, 2004, available on-line at www.ub.rug.nl/eldoc/dis/science/ f.p.mena

${ }^{33}$ T. Jarlborg, Phys. Rev. B 59, 15002 (1999).

${ }^{34}$ K. Urasaki and T. Saso, Physica B 281-282, 313 (1999).

${ }^{35}$ K. Urasaki and T. Saso, Phys. Rev. B 58, 15528 (1998).

${ }^{36}$ M. J. Rozenberg, G. Kotliar, and H. Kajueter, Phys. Rev. B 54, 8452 (1996).

${ }^{37}$ C. Fu, M. P. C. M. Krijn, and S. Doniach, Phys. Rev. B 49, 2219 (1994).

${ }^{38}$ M. A. Chernikov, L. Degiorgi, E. Felder, S. Paschen, A. D. Bianchi, H. R. Ott, J. L. Sarrao, Z. Fisk, and D. Mandrus, Phys. Rev. B 56, 1366 (1997).

${ }^{39}$ J. Guevara, V. Vildosola, J. Milano, and A. M. Llois, Phys. Rev. B 69, 184422 (2004).

${ }^{40}$ P. A. Wolff, Phys. Rev. 124, 1030 (1961).

${ }^{41}$ A. M. Clogston, B. T. Matthias, M. Peter, H. J. Williams, E. Corenzwit, and R. C. Sherwood, Phys. Rev. 125, 541 (1962).

${ }^{42}$ P. W. Anderson, Phys. Rev. 124, 41 (1961).

${ }^{43}$ P. W. Anderson, Rev. Mod. Phys. 50, 191 (1978).

${ }^{44}$ O. Gunnarsson and B. Lundqvist, Phys. Rev. B 13, 4274 (1976).

${ }^{45}$ S. Y. Savrasov and D. Y. Savrasov, Phys. Rev. B 46, 12181 (1992).

${ }^{46}$ I. I. Mazin, E. G. Maksimov, S. N. Rashkeev, S. Yu. Savrasov, and Yu. A. Uspenskii, JETP Lett. 47, 113 (1988).

${ }^{47}$ L. F. Mattheiss and D. R. Hamann, Phys. Rev. B 47, 13114 (1993).

${ }^{48}$ A. Fert and A. Campbell, Phys. Rev. Lett. 21, 1190 (1968). 